\title{
Prognostic value of alkaline phosphatase in hormone-sensitive prostate cancer: a systematic review and meta-analysis
}

\author{
Keiichiro Mori ${ }^{1,2}$ (1) Florian Janisch ${ }^{1,3} \cdot$ Mehdi Kardoust Parizi $^{1,4} \cdot$ Hadi Mostafaei ${ }^{1,5} \cdot$ Ivan Lysenko $^{1}$. \\ Dmitry V. Enikeev ${ }^{10}$. Shoji Kimura ${ }^{1,2} \cdot$ Shin Egawa ${ }^{2} \cdot$ Shahrokh F. Shariat ${ }^{1,6,7,8,9,10}$
}

Received: 8 October 2019 / Accepted: 7 November 2019 / Published online: 25 November 2019

(c) The Author(s) 2019

\begin{abstract}
Purpose To assess the prognostic value of alkaline phosphatase in patients with hormone-sensitive prostate cancer.

Methods A systematic review and meta-analysis was performed using the PUBMED, Web of Science, Cochrane Library, and Scopus in April 2019 according to the Preferred Reporting Items for Systematic Review and Meta-analysis statement. Studies were deemed eligible if they compared hormone-sensitive prostate cancer patients with high vs. low alkaline phosphatase to determine its predictive value for overall survival, cancer-specific survival, and progression-free survival. We performed a formal meta-analysis of these outcomes.

Results 42 articles with 7938 patients were included in the systematic review and 28 studies with 5849 patients for the qualitative assessment. High alkaline phosphatase was associated with worse overall survival (pooled HR 1.72; 95\% CI 1.37-2.14) and progression-free survival (pooled HR 1.30; 95\% CI 1.10-1.54). In subgroup analyses of patients with "high-volume" and "low-volume", alkaline phosphatase was associated with the overall survival (pooled HR 1.41; 95\% CI 1.21-1.64 and pooled HR 1.64; 95\% CI, 1.06-2.52, respectively).

Conclusions In this meta-analysis, elevated serum levels of alkaline phosphatase were associated with an increased risk of overall mortality and disease progression in patients with hormone-sensitive prostate cancer. In contrast, those were not associated with an increased risk of cancer-specific mortality. Alkaline phosphatase was independently associated with overall survival in both patients with "high-volume" and "low-volume" hormone-sensitive prostate cancer. Alkaline phosphatase may be useful for being integrated into prognostic tools that help guide treatment strategy, thereby facilitating the shared decision making process.
\end{abstract}

Keywords Alkaline phosphatase (ALP) · Hormone-sensitive prostate cancer (HSPC) · Meta-analysis

Shahrokh F. Shariat

shahrokh.shariat@meduriwien.ac.at

1 Department of Urology, Medical University of Vienna, Währinger Gürtel 18-20, 1090 Vienna, Austria

2 Department of Urology, The Jikei University School of Medicine, Tokyo, Japan

3 Department of Urology, Medical University of Hamburg, Hamburg, Germany

4 Department of Urology, Shariati Hospital, Tehran University of Medical Sciences, Teheran, Iran

5 Research Center for Evidence Based Medicine, Tabriz University of Medical Sciences, Tabriz, Iran
6 Department of Urology, Weill Cornell Medical College, New York, NY, USA

7 Department of Urology, University of Texas Southwestern, Dallas, TX, USA

8 Karl Landsteiner Institute of Urology and Andrology, Vienna, Austria

9 Department of Urology, Second Faculty of Medicine, Charles University, Prague, Czech Republic

10 Institute for Urology and Reproductive Health, I.M. Sechenov First Moscow State Medical University, Moscow, Russia 


\section{Introduction}

Prostate cancer (PC) is not only the most common solid cancer, but also the second most common cause of cancer-related death in men [1]. Following the results of the CHAARTED trial and the LATITUDE trial, the treatment of patients with metastatic hormone-sensitive prostate cancer (HSPC) has changed substantially in the recent years $[2,3]$. However, systemic therapy based on androgen deprivation remains the standard primary treatment strategy in patients with metastatic HSPC. Despite adequate therapy, the disease eventually progresses to a castration-resistant prostate cancer (CRPC) [4]. To improve PC outcomes, prognostic tools have been developed to help in the daily clinical decision making and patient counselling [5-8]. These tools include standard clinical features and biomarkers [9], such as alkaline phosphatase (ALP) in patients with CRPC, but not yet in patients with HSPC.

ALP is a glycoprotein derived from bones, liver, kidney, or placenta that has been shown to be elevated and of prognostic value for various malignancies [10-13]. In PC, ALP has been shown to be of prognostic value in CRPCreflecting disease outcome, independent of therapy [14]. In patients with CRPC, high-baseline ALP levels have been shown to be associated with worse outcomes, including skeletal complications and decreased survival [15-17]. Moreover, elevated ALP was also been shown to be correlated with the extent of metastatic bone disease [17, 18]. Serum ALP is deemed a simple and inexpensive test that could serve as an objective prognostic parameter that helps improve daily oncologic clinical practice, plan follow-up, and counsel regarding outcomes, thus facilitating the shared decision making process with the patient. Unfortunately, to date, the prognostic value of ALP in HSPC remains insufficiently investigated.

The aim of the current study was to summarize the available data to test the hypothesis that ALP has a strong prognostic value for oncologic outcomes in HSPC patients. To this end, we performed a systematic review and a meta-analysis.

\section{Materials and methods}

\section{Search strategy}

This systematic review and meta-analysis was performed according to the Preferred Reporting Items for Systematic Reviews and Meta-analyses (PRISMA) statement [19]. We searched the electronic databases PUBMED, Web of Science, Cochrane Library and Scopus on April 2019, investigating the prognostic value of ALP in HSPC.
After the first screening based on study title and abstract, all papers were assessed based on full text and excluded with reasons when inappropriate; a further check of the appropriateness of the papers based on full text revision which was performed after the data extraction. Two investigators carried out this process independently. Disagreements were resolved by a consensus meeting with a third investigator. The following keywords were used in our search strategy: (prostate cancer OR prostate carcinoma OR prostate tumor OR prostatic carcinoma OR prostatic cancer OR prostatic tumor NOT resistant) AND (Alkaline Phosphatase OR ALP) AND (survival OR outcome OR prognostic OR mortality OR progression OR recurrence OR OS OR CSS OR PFS OR RFS OR MFS). The primary outcome of interest was overall survival (OS) and secondary outcomes were cancer-specific survival (CSS) and progression-free survival (PFS).

\section{Inclusion criteria and exclusion criteria}

Studies were included if they investigated whether patients with high ALP treated for HSPC (patients) who had received systemic therapy (intervention) as compared to those who had low ALP (comparison) to assess the independent predictive value of ALP on OS, CSS, and PFS (outcome) utilizing multivariate Cox regression analysis (study design) in nonrandomized observational, or randomize or cohort studies. We excluded reviews, letters, editorials, meeting abstracts, replies from author, case reports, and articles not published in English. In case of duplicate publications, either the higher quality or the most recent publication was selected. References of included manuscripts were scanned for additional studies of interest.

\section{Data extraction}

Two investigators independently extracted the information from the included articles. The information contained the following characteristics: first author's name, publication year, recruitment country, period of patient recruitment, number of patients, age, study design, disease stage, therapy type, oncological outcome, follow-up duration, conclusion, and ALP cut-off. Subsequently, the hazard ratios (HR) and 95\% confidence intervals (CI) of ALP associated with each of the outcomes were retrieved. The HRs were extracted from the multivariate analyses. All discrepancies regarding data extraction were resolved by consensus with a third investigator.

\section{Quality assessment}

The Newcastle-Ottawa Scale (NOS) was used to assess the quality of the included studies according to the Cochrane 
Handbook for systematic reviews of interventions for included non-randomized studies [20,21]. The scale focuses on the three factors: Selection (1-4), Comparability (1-2) and Exposure (1-3). The total score ranges from 0 (lowest) to 9 (highest). The main confounders were identified as the important prognostic factors of OS, CSS, and PFS. The presence of confounders was determined by consensus and review of the literature. We identified as "high-quality" choices those with scores more than 6 .

\section{Statistical analyses}

We performed a forest plot to assess the HRs from the multivariate analyses of individual studies and obtained a summary HR of the value of ALP on OS, CSS, and PFS. Disease progression includes symptomatic or radiographic or biochemical progression in this analysis. Studies with Kaplan-Meier log-rank, univariate Cox proportional hazard regression, or general logistic regression analyses were not considered for the meta-analysis. In case there were only HR and $P$ value, we calculated $95 \%$ CI $[22,23]$. We also performed subgroup analyses in HSPC patients with "high-volume" and "low-volume" disease. We classified as low-volume (lesions $<4$ sites and within pelvis-vertebral column) or high-volume disease (lesions $\geq 4$ sites and at least one lesion beyond the pelvis-vertebral column) according to the CHAARTED classification [2]. Again, of all the HSPC patients from the studies providing information on EOD scores or Soloway scores, those with EOD scores 2 or higher or those with Soloway scores 2 or higher were defined as high-volume disease [24]. With high-volume disease thus defined, all studies in which those with high-volume disease accounted for $60 \%$ or more or less than $60 \%$ of all patients were included for the current analysis as "high-volume disease" and "low-volume disease" studies, respectively.

Heterogeneity among the outcomes of the included studies in this meta-analysis was evaluated using Cochrane $Q$ test and $I^{2}$ statistic. Significant heterogeneity was indicated by a $P<0.05$ in Cochrane $Q$ tests and a ratio $>50 \%$ in $I^{2}$ statistics, which led to the use of random-effect models. We used fixed effect models for calculation of pooled HRs for nonheterogeneous results [25-27]. Publication bias was assessed by funnel plots. Statistical analyses were performed using Stata/MP 14.2 (Stata Corp., College Station, TX); statistical significance level was set at $P<0.05$.

\section{Results}

\section{Study selection and characteristics}

Our initial search identified 2245 records. After removal of duplicates, 2016 remained (Fig. 1). After screening of the titles and abstracts, 1816 articles were excluded. Then we assessed 200 full texts for further selection. After selection, 42 articles with 7938 patients were included in the systematic review and 28 studies with 5849 patients for qualitative meta-analysis [28-69]. The baseline characteristics of the 42 studies are outlined in Table 1. All included studies were published between 1995 and 2019 with 15 being from Europe, and 27 from Asia. Median age ranged from 63 to 77 years, 10 studies included non-metastatic HSPC. Studies were heterogeneous regarding cut-off value for ALP ranging from 67 to 620 for OS, from 115 to 683.4 for CSS, and from 114.56 to 400 for PFS; follow-up ranged from 14.4 to 156 months.

\section{Meta-analysis}

\section{Association of ALP with OS in HSPC}

Sixteen studies including 3747 patients provided data on the association of ALP with OS in HSPC. The forest plot (Fig. 2a) showed that ALP was significantly associated with OS in HSPC (pooled HR 1.72; 95\% CI 1.37-2.14; $z=4.76$ ). The Cochrane $Q$ test $\left(\chi^{2}=85.73 ; P=0.000\right)$ and $I^{2}$ test $\left(I^{2}=81.3 \%\right)$ showed significant heterogeneity. The funnel plot identified nine studies over the pseudo 95\% CI (Fig. 2a).

\section{Association of ALP with CSS in HSPC}

Ten studies including 2225 patients provided data on the association of ALP with CSS in HSPC. The forest plot (Fig. 2b) showed that ALP was not significantly associated with CSS in HSPC (pooled HR 1.00; 95\% CI 1.00-1.01; $z=1.55)$. The Cochrane $Q$ test $\left(\chi^{2}=80.97 ; P=0.000\right)$ and $I^{2}$ test $\left(I^{2}=88.9 \%\right)$ showed significant heterogeneity. The funnel plot identified four studies over the pseudo $95 \%$ CI (Fig. 2b).

\section{Association of ALP with PFS in HSPC}

Seven studies including 1547 patients provided data on the association of ALP with PFS in HSPC. The forest plot (Fig. 2c) showed that ALP was significantly associated with PFS in HSPC (pooled HR 1.30; 95\% CI 1.10-1.54; $z=3.04)$. The Cochrane $Q$ test $\left(\chi^{2}=40.49 ; P=0.000\right)$ and $I^{2}$ test $\left(I^{2}=85.2 \%\right)$ showed significant heterogeneity. The funnel plot identified four studies over the pseudo $95 \%$ CI (Fig. 2c).

\section{Association of ALP with OS in HSPC with "high volume"}

Five studies including 1509 patients provided data on the association of ALP with OS in HSPC with "high-volume" disease. The forest plot (Fig. 3a) showed that ALP was 


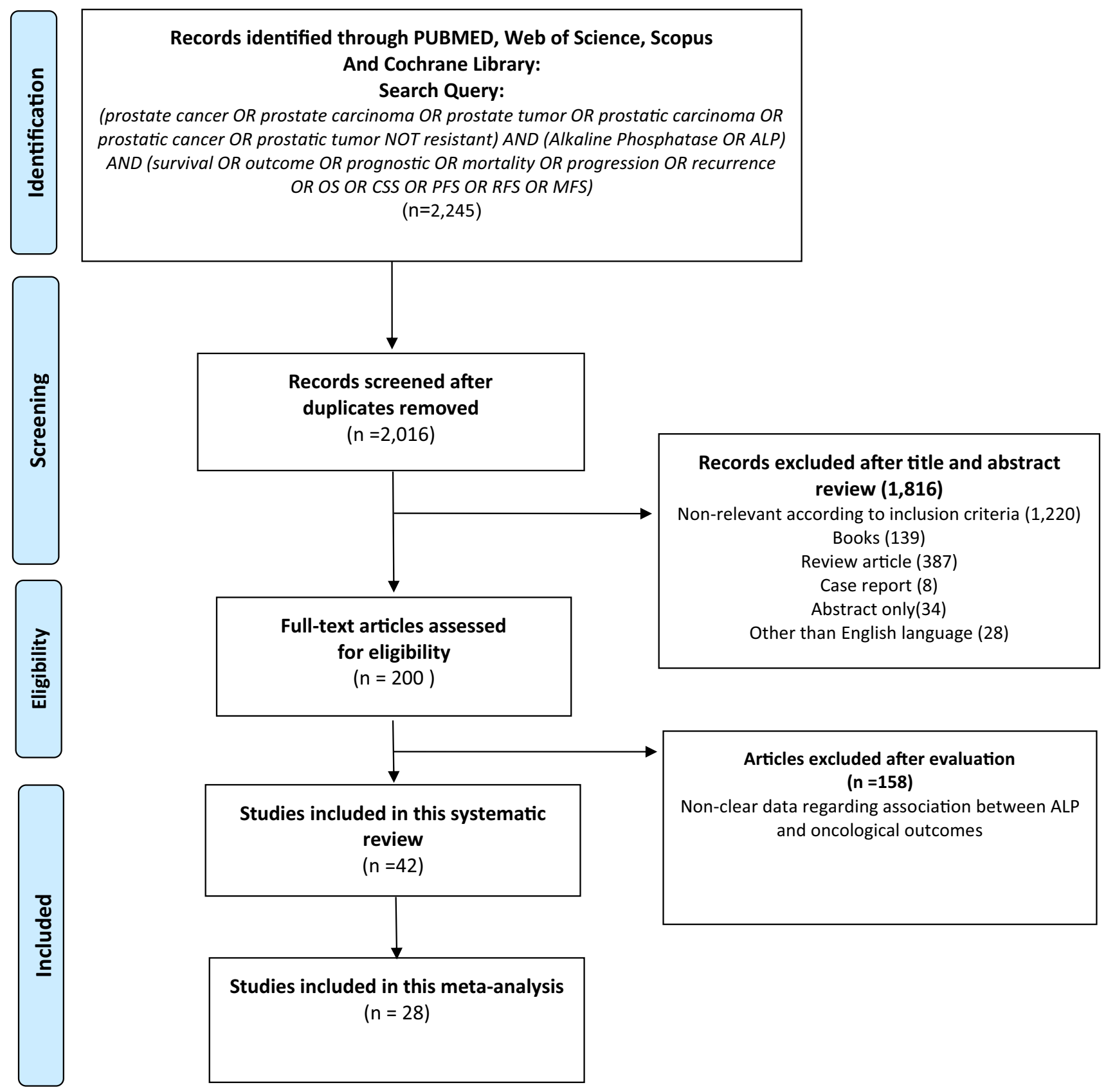

Fig. 1 The Preferred Reporting Items for Systematic Reviews and Meta-analyses (PRISMA) flow chart for article selection process to analyze the prognostic value of alkaline phosphatase (ALP) in hormone-sensitive prostate cancer (HSPC) and oncological outcomes

significantly associated with OS in HSPC with "highvolume" disease (pooled HR 1.41; 95\% CI 1.21-1.64; $z=4.47)$. The Cochrane $Q$ test $\left(\chi^{2}=7.25 ; P=0.123\right)$ and $I^{2}$ test $\left(I^{2}=44.8 \%\right)$ showed no significant heterogeneity. The funnel plot identified no studies over the pseudo $95 \%$ CI (Fig. 3a).

\section{Association of ALP with OS in HSPC with "low volume"}

Six studies including 1039 patients provided data on the association of ALP with OS in HSPC with "low-volume" disease. The forest plot (Fig. 3b) showed that ALP was significantly associated with OS in HSPC with "low-volume" 


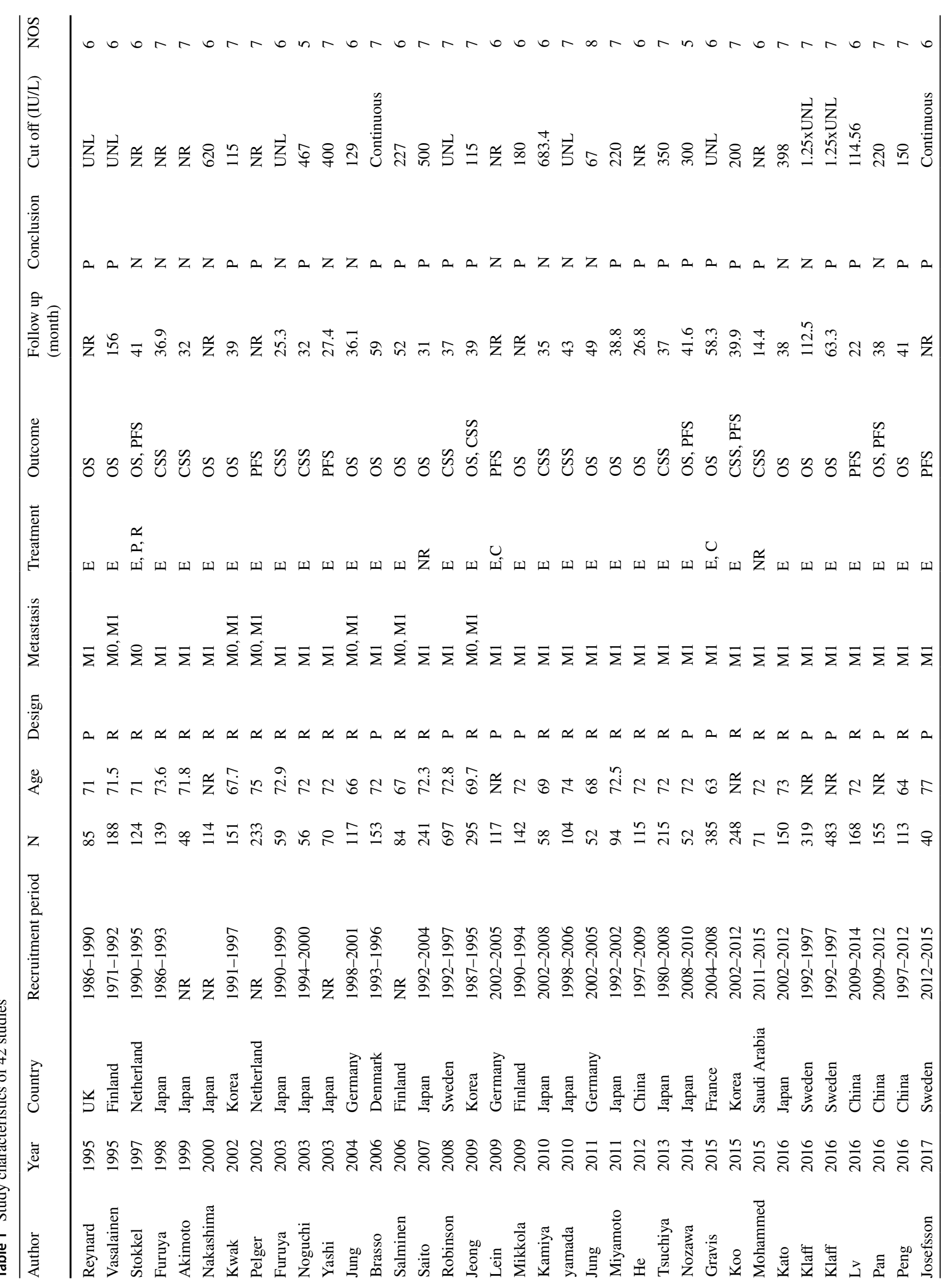




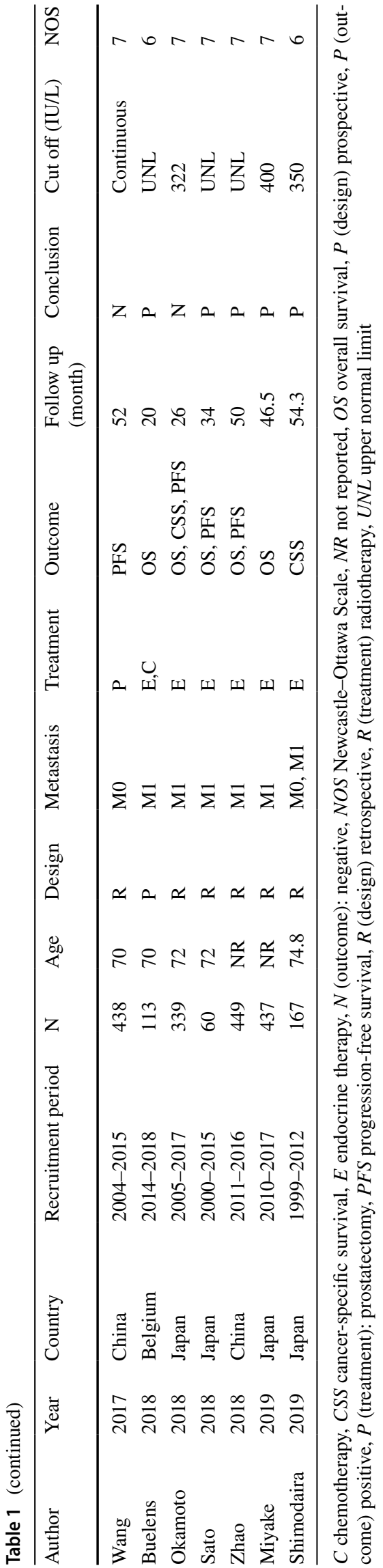

disease (pooled HR 1.64; 95\% CI 1.06-2.52; $z=2.25$ ). The Cochrane $Q$ test $\left(\chi^{2}=22.10 ; P=0.001\right)$ and $I^{2}$ test $\left(I^{2}=77.4 \%\right)$ showed significant heterogeneity. The funnel plot identified three studies over the pseudo 95\% CI (Fig. 3b).

\section{Discussion}

In this systematic review and meta-analysis, we investigated the prognostic value of ALP in HSPC by assessing its impact on PFS, CSS, and OS. We found that the HSPC patients with elevated ALP have significantly worse OS and PFS compared to their counterparts with normal ALP levels. In other words, pre-treatment ALP values may be a useful biomarker in the choice of treatment, even in early metastatic PC.

The prognostic value of ALP has been shown in various solid malignancies with bone metastasis [11-13]. However, while there is a biological rationale underlying this association, the exact mechanism remains unclear. A potential explanation is that when cancer starts to metastasize, ALP reflects bone turnover, osteoblast activity, and osteoid formation in the adjacent bone tissues [70]. Thus, ALP may be an indicator of bone metastatic tumor load. Accordingly, ALP has been shown to be elevated in cancer patients with bone metastasis, as the current literature shows, ALP is already among the biomarkers included in the tools used for prognosticating outcomes in CRPC patients [5-8].

Interestingly, ALP was significantly associated with worse OS in metastatic HSPC patients not only with "high-volume" disease, but also with "low-volume" disease, suggesting that ALP is an indirect sensitive measure of metastatic tumor burden which could not be captured by conventional imaging. It is likely that the elevated ALP reflects micro metastases despite negative findings on conventional imaging. Although few studies have assessed this patient subgroup, ALP could be used to select patients who may benefit more from intensive therapy such as upfront docetaxel or abiraterone in addition to standard androgen deprivation therapy. Moreover, ALP could also be used as a response/monitoring marker for these therapies as well as bone-targeting therapies such as bisphosphonate.

Despite showing a strong association of ALP with mortality and progression in HSPC patients, this systematic review and meta-analysis has some limitations. There is a reporting bias, as some studies with negative results may not have been published. Further, many included studies were retrospective, leading to a patient selection bias. Second, unknown pretreatment conditions (i.e., physical conditions, comorbidities, obstructive jaundice, bone disease, hyperthyroidism and hepatitis, medication, and lifestyle habits) could have altered ALP values leading to a 
(A)

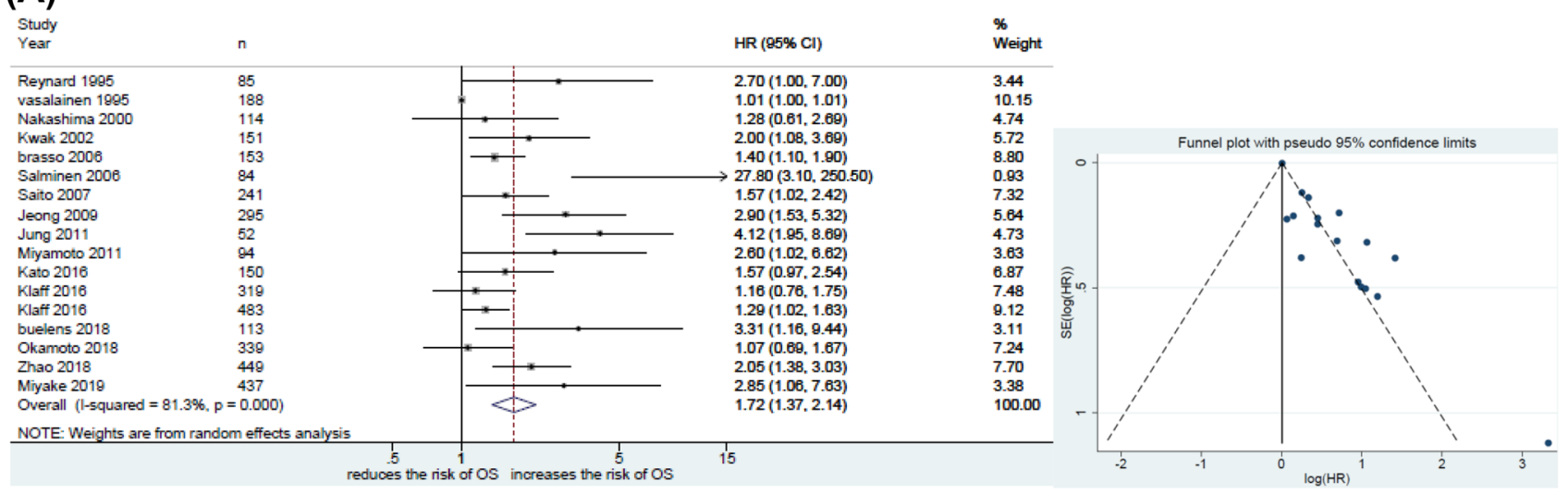

(B)

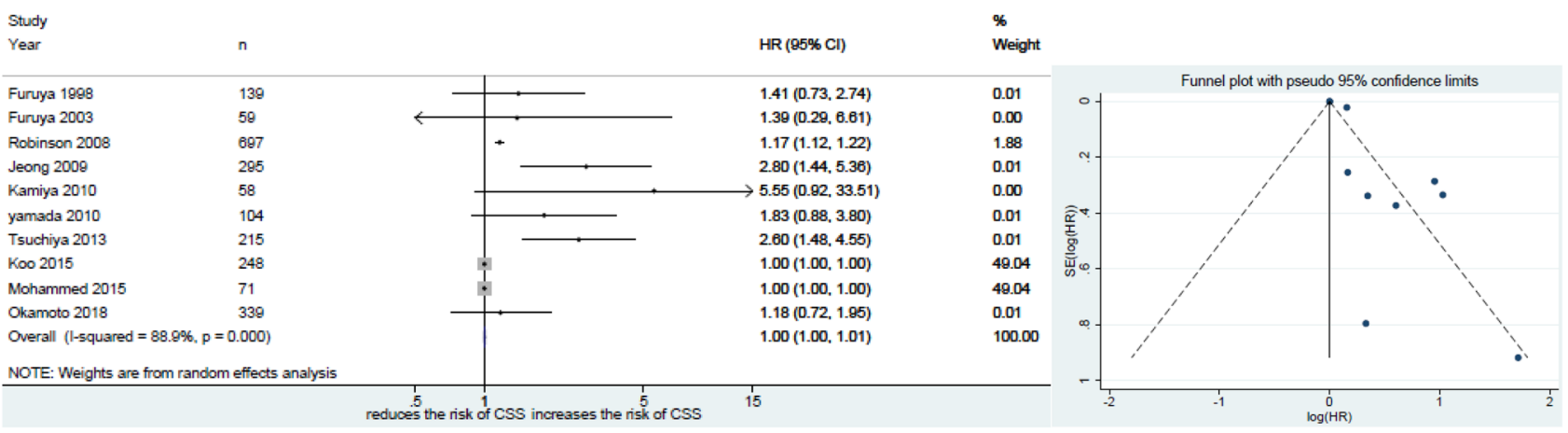

(C)

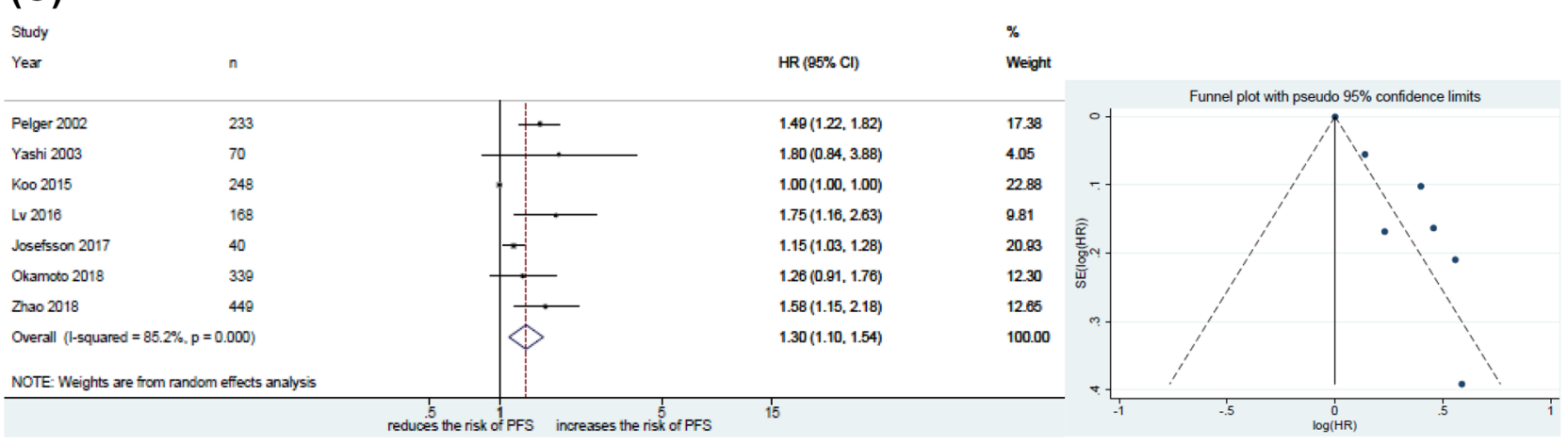

Fig. 2 Forest and funnel plots showing the association of alkaline phosphatase (ALP) with oncologic outcomes in hormone-sensitive prostate cancer (HSPC): a overall survival b cancer specific surivival (C) progression free survival

systematic bias. Third, heterogeneity was detected for OS, CSS, and PFS analyses limiting the value of these results. Although the random effect model takes into account the heterogeneity among studies, the conclusions should be interpreted with caution. Fourth, there is no established cut-off value for ALP among the included studies; most investigators chose the cut-off based on the statistical methods assessing for the highest sensitivity and specificity, using the upper limit of normal, or using literature predefined ALP cut-offs. Only three studies investigated ALP as a continuous variable. Regardless of these limitations, ALP is a fast and readily available biomarker. Well-designed prospective studies with longer follow-up are needed to validate the prognostic value of ALP and its potential value in risk stratification of patients with HSPC using clinical decision-analytical tools. 


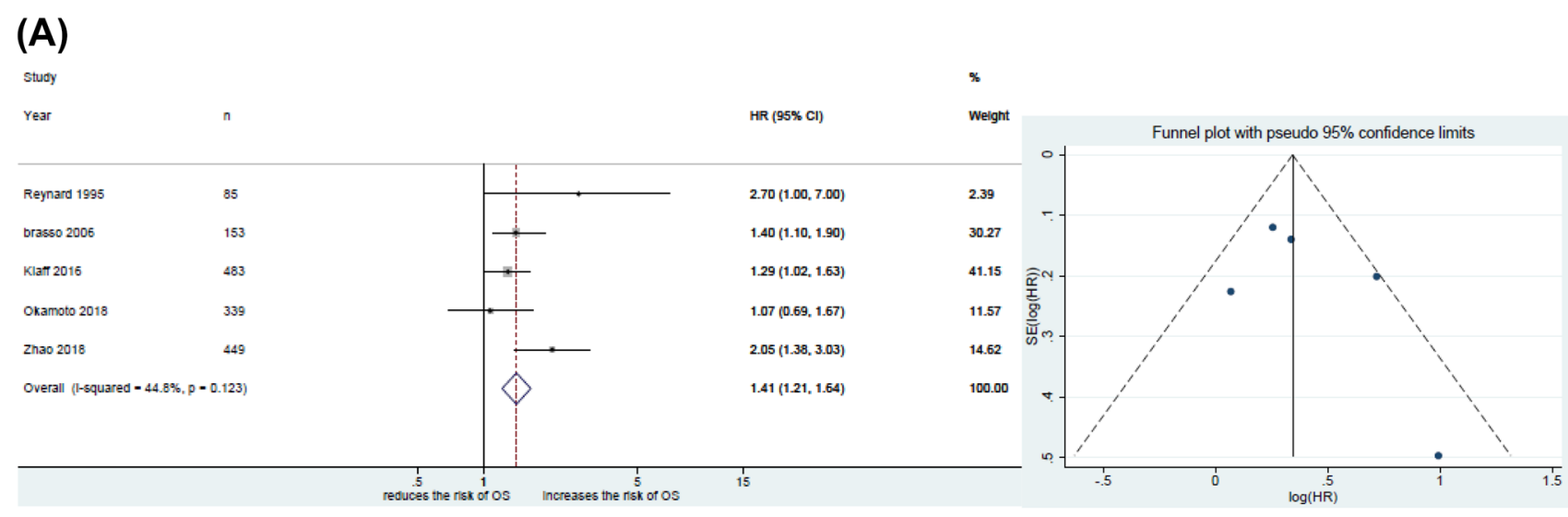

\section{(B)}

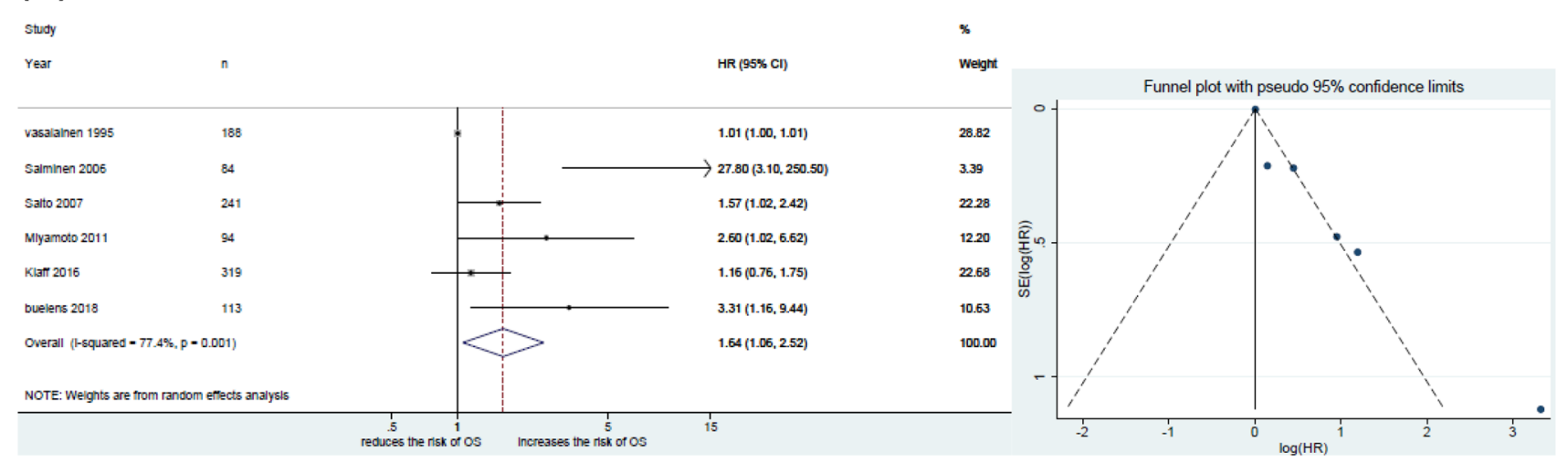

Fig. 3 Forest and funnel plots showing the association of alkaline phosphatase (ALP) with oncologic outcomes: a overall survival in hormone-sensitive prostate cancer (HSPC) with "high-volume" dis- ease b overall survival in hormone-sensitive prostate cancer (HSPC) with "low-volume" disease

\section{Conclusions}

In this meta-analysis, high serum ALP was associated with an increased risk of overall mortality and disease progression in patients with HSPC. In contrast, high serum ALP was not associated with an increased risk of cancer-specific mortality. Furthermore, ALP was an independent risk factor for OS in HSPC patients with both "high-volume" and "low-volume" metastatic disease. ALP may be useful for clinical decision making regarding treatment selection, as well as for patient counselling. However, considering the limitations including heterogeneity, the conclusions should be interpreted with caution.

Acknowledgements Open access funding provided by Medical University of Vienna.

Author contributions Project development: KM, FJ, DVE, SK, SE, SFS. Data collection: KM, FJ. Data analysis: KM, FJ. Manuscript writing/editing: KM, FJ, MKP, HM, IL, DVE, SK, SE, SFS.
Funding This research did not receive any specific grant from funding agencies in the public, commercial, or not-for-profit sectors.

\section{Compliance with ethical standards}

Conflict of interest None of the authors have conflicts of interest to disclose.

Open Access This article is distributed under the terms of the Creative Commons Attribution 4.0 International License (http://creativeco mmons.org/licenses/by/4.0/), which permits unrestricted use, distribution, and reproduction in any medium, provided you give appropriate credit to the original author(s) and the source, provide a link to the Creative Commons license, and indicate if changes were made.

\section{References}

1. Siegel RL, Miller KD, Jemal A (2018) Cancer statistics, 2018. CA Cancer J Clin 68(1):7-30. doi:10.3322/caac.21442

2. Sweeney CJ, Chen YH, Carducci M et al (2015) Chemohormonal therapy in metastatic hormone-sensitive prostate cancer. 
New Engl J Med 373(8):737-746. https://doi.org/10.1056/ NEJMoa1503747

3. Fizazi K, Tran N, Fein L et al (2017) Abiraterone plus prednisone in metastatic, castration-sensitive prostate cancer. New Engl J Med 377(4):352-360. https://doi.org/10.1056/NEJMoa1704174

4. Kluth LA, Shariat SF, Kratzik C et al (2014) The hypothalamicpituitary-gonadal axis and prostate cancer: implications for androgen deprivation therapy. World J Urol 32(3):669-676. https://doi.org/10.1007/s00345-013-1157-5

5. Chi KN, Kheoh T, Ryan CJ et al (2016) A prognostic index model for predicting overall survival in patients with metastatic castration-resistant prostate cancer treated with abiraterone acetate after docetaxel. Ann Oncol 27(3):454-460. https://doi. org/10.1093/annonc/mdv594

6. Halabi S, Lin CY, Kelly WK et al (2014) Updated prognostic model for predicting overall survival in first-line chemotherapy for patients with metastatic castration-resistant prostate cancer. J Clin Oncol 32(7):671-677. https://doi.org/10.1200/ jco.2013.52.3696

7. Armstrong AJ, Garrett-Mayer E, de Wit R et al (2010) Prediction of survival following first-line chemotherapy in men with castration-resistant metastatic prostate cancer. Clin Cancer Res 16(1):203-211. https://doi.org/10.1158/1078-0432.ccr-09-2514

8. Shariat SF, Kattan MW, Vickers AJ et al (2009) Critical review of prostate cancer predictive tools. Fut Oncol (Lond Engl) 5(10):1555-1584. https://doi.org/10.2217/fon.09.121

9. Shariat SF, Semjonow A, Lilja $\mathrm{H}$ et al (2011) Tumor markers in prostate cancer I: blood-based markers. Acta Oncol (Stockholm, Sweden) 50(Suppl 1):61-75. https://doi.org/10.3109/02841 86x.2010.542174

10. Pochanugool L, Subhadharaphandou T, Dhanachai M et al (1997) Prognostic factors among 130 patients with osteosarcoma. Clin Orthop Relat Res 345:206-214

11. Liu F, Zhao J, Xie J et al (2016) Prognostic risk factors in patients with bone metastasis from colorectal cancer. Tumour Biol. https ://doi.org/10.1007/s13277-016-5465-4

12. Lim SM, Kim YN, Park KH et al (2016) Bone alkaline phosphatase as a surrogate marker of bone metastasis in gastric cancer patients. BMC Cancer 16:385. https://doi.org/10.1186/s12885-016-2415-x

13. Huang P, Lan M, Peng AF et al (2017) Serum calcium, alkaline phosphotase and hemoglobin as risk factors for bone metastases in bladder cancer. PLoS ONE 12(9):e0183835. https://doi. org/10.1371/journal.pone.0183835

14. Bitting RL, Armstrong AJ (2013) Potential predictive biomarkers for individualizing treatment for men with castration-resistant prostate cancer. Cancer J (Sudbury, Mass) 19(1):25-33. https:// doi.org/10.1097/PPO.0b013e31827e0b9c

15. Brown JE, Cook RJ, Major P et al (2005) Bone turnover markers as predictors of skeletal complications in prostate cancer, lung cancer, and other solid tumors. J Natl Cancer Inst 97(1):59-69. https://doi.org/10.1093/jnci/dji002

16. Coleman RE, Major P, Lipton A et al (2005) Predictive value of bone resorption and formation markers in cancer patients with bone metastases receiving the bisphosphonate zoledronic acid. J Clin Oncol 23(22):4925-4935. https://doi.org/10.1200/ jco.2005.06.091

17. Armstrong AJ, Febbo PG (2009) Using surrogate biomarkers to predict clinical benefit in men with castration-resistant prostate cancer: an update and review of the literature. Oncologist 14(8):816-827. https://doi.org/10.1634/theoncologist.2009-0043

18. D'Oronzo S, Brown J, Coleman R (2017) The value of biomarkers in bone metastasis. Eur J Cancer Care (Engl). doi:10.1111/ecc.12725

19. Liberati A, Altman DG, Tetzlaff J et al (2009) The PRISMA statement for reporting systematic reviews and meta-analyses of studies that evaluate health care interventions: explanation and elaboration. PLoS Med 6(7):e1000100. https://doi.org/10.1371/ journal.pmed.1000100

20. Stang A (2010) Critical evaluation of the Newcastle-Ottawa scale for the assessment of the quality of nonrandomized studies in meta-analyses. Eur J Epidemiol 25(9):603-605. https://doi. org/10.1007/s10654-010-9491-z

21. Deeks JJ, Dinnes J, D’Amico R et al (2003) Evaluating non-randomised intervention studies. Health Technol Assess (Winchester, England) 7(27):iii-x, 1-173

22. Altman DG, Bland JM (2011) How to obtain the confidence interval from a P value. BMJ (Clin Res) 343:d2090. https://doi. org/10.1136/bmj.d2090

23. Altman DG, Bland JM (2011) How to obtain the P value from a confidence interval. BMJ (Clin Res) 343:d2304. https://doi. org/10.1136/bmj.d2304

24. Soloway MS, Hardeman SW, Hickey D et al (1988) Stratification of patients with metastatic prostate cancer based on extent of disease on initial bone scan. Cancer 61(1):195-202

25. DerSimonian R, Kacker R (2007) Random-effects model for meta-analysis of clinical trials: an update. Contemp Clin Trial 28(2):105-114. https://doi.org/10.1016/j.cct.2006.04.004

26. DerSimonian R, Laird N (1986) Meta-analysis in clinical trials. Control Clin Trials 7(3):177-188

27. Higgins JP, Thompson SG, Deeks JJ et al (2003) Measuring inconsistency in meta-analyses. BMJ 327(7414):557-560. https://doi. org/10.1136/bmj.327.7414.557

28. Akimoto S, Furuya Y, Akakura K et al (1999) Inability of bone turnover marker as a strong prognostic indicator in prostate cancer patients with bone metastasis: comparison with the extent of disease (EOD) grade. Prostate 38(1):28-34

29. Brasso K, Christensen IJ, Johansen JS et al (2006) Prognostic value of PINP, bone alkaline phosphatase, CTX-I, and YKL-40 in patients with metastatic prostate carcinoma. Prostate 66(5):503513. https://doi.org/10.1002/pros.20311

30. Buelens S, De Bleser E, Dhondt B et al (2018) Importance of metastatic volume in prognostic models to predict survival in newly diagnosed metastatic prostate cancer. World J Urol. https ://doi.org/10.1007/s00345-018-2449-6

31. Furuya Y, Akimoto S, Akakura K (1998) Response of prostatespecific antigen after androgen withdrawal and prognosis in men with metastatic prostate cancer. Urol Int 60(1):28-32. https://doi. org/10.1159/000030199

32. Furuya Y, Nagakawa O, Fuse H (2003) Prognostic significance of changes in short-term prostate volume and serum prostate-specific antigen after androgen withdrawal in men with metastatic prostate cancer. Urol Int 70(3):195-199. https://doi.org/10.1159/00006 8769

33. Gravis G, Boher JM, Fizazi K et al (2015) Prognostic factors for survival in noncastrate metastatic prostate cancer: validation of the glass model and development of a novel simplified prognostic model. Eur Urol 68(2):196-204. https://doi.org/10.1016/j.eurur o.2014.09.022

34. He J, Zeng ZC, Yang P et al (2012) Clinical features and prognostic factors for patients with bone metastases from prostate cancer. Asian J Androl 14(3):505-508. https://doi.org/10.1038/ aja.2012.24

35. Jeong CW, Ku JH, Kwak C et al (2009) Chronic pulmonary disease negatively influences the prognosis of patients with advanced prostate cancer. World J Urol 27(5):643-652. https:// doi.org/10.1007/s00345-009-0375-3

36. Josefsson A, Linder A, Flondell Site D et al (2017) Circulating tumor cells as a marker for progression-free survival in metastatic castration-naïve prostate cancer. Prostate 77(8):849-858. https:// doi.org/10.1002/pros.23325

37. Jung K, Lein M, Stephan C et al (2004) Comparison of 10 serum bone turnover markers in prostate carcinoma patients with bone 
metastatic spread: diagnostic and prognostic implications. Int $\mathrm{J}$ Cancer 111(5):783-791. https://doi.org/10.1002/ijc.20314

38. Jung K, Miller K, Wirth M et al (2011) Bone turnover markers as predictors of mortality risk in prostate cancer patients with bone metastases following treatment with zoledronic acid. Eur Urol 59(4):604-612. https://doi.org/10.1016/j.eururo.2010.12.006

39. Kamiya N, Suzuki H, Yano M et al (2010) Implications of serum bone turnover markers in prostate cancer patients with bone metastasis. Urology 75(6):1446-1451. https://doi.org/10.1016/j. urology.2009.11.049

40. Kato M, Tsuzuki T, Kimura K et al (2016) The presence of intraductal carcinoma of the prostate in needle biopsy is a significant prognostic factor for prostate cancer patients with distant metastasis at initial presentation. Modern Pathol 29 (2):166-173. doi:10.1038/modpathol.2015.146

41. Klaff R, Varenhorst E, Berglund A et al (2016) Clinical presentation and predictors of survival related to extent of bone metastasis in 900 prostate cancer patients. Scand J Urol 50(5):352-359. https ://doi.org/10.1080/21681805.2016.1209689

42. Koo KC, Park SU, Kim KH et al (2015) Predictors of survival in prostate cancer patients with bone metastasis and extremely high prostate-specific antigen levels. Prostate Int 3(1):10-15. https:// doi.org/10.1016/j.prnil.2015.02.006

43. Kwak C, Jeong SJ, Park MS et al (2002) Prognostic significance of the nadir prostate specific antigen level after hormone therapy for prostate cancer. J Urol 168(3):995-1000. https://doi. org/10.1097/01.ju.0000024925.67014.21

44. Lein M, Miller K, Wirth M et al (2009) Bone Turnover markers as predictive tools for skeletal complications in men with metastatic prostate cancer treated with zoledronic acid. Prostate 69(6):624632. https://doi.org/10.1002/pros.20917

45. Lv W, Shang HX, Pei XQ et al (2017) A simple prognostic model involving prostate-specific antigen, alkaline phosphatase and albumin for predicting the time required to progress to castration-resistant prostate cancer in patients who received androgen deprivation therapy. Int Urol Nephrol 49(1):61-67. https://doi. org/10.1007/s11255-016-1456-Z

46. Mikkola A, Aro J, Rannikko S et al (2009) Prognostic grouping of metastatic prostate cancer using conventional pretreatment prognostic factors. Scand J Urol Nephrol 43(4):265-270. https:// doi.org/10.1080/00365590902836500

47. Miyake H, Matsushita Y, Watanabe H et al (2019) Prognostic significance of time to castration resistance in patients with metastatic castration-sensitive prostate cancer. Anticancer Res 39(3):1391-1396. https://doi.org/10.21873/anticanres.13253

48. Miyamoto S, Ito K, Miyakubo M et al (2012) Impact of pretreatment factors, biopsy Gleason grade volume indices and post-treatment nadir PSA on overall survival in patients with metastatic prostate cancer treated with step-up hormonal therapy. Prostate Cancer Prostatic Dis 15(1):75-86. https://doi.org/10.1038/pcan.2011.47

49. Mohammed AA, El-Tanni H, Ghanem HM et al (2015) Impact of body mass index on clinico-pathological parameters and outcome in patients with metastatic prostate cancer. JEgypt Natl Cancer Inst 27(3):155-159. https://doi.org/10.1016/j.jnci.2015.07.001

50. Nakashima J, Ozu C, Nishiyama T et al (2000) Prognostic value of alkaline phosphatase flare in patients with metastatic prostate cancer treated with endocrine therapy. Urology 56(5):843-847

51. Noguchi M, Kikuchi H, Ishibashi M et al (2003) Percentage of the positive area of bone metastasis is an independent predictor of disease death in advanced prostate cancer. Br J Cancer 88(2):195201. https://doi.org/10.1038/sj.bjc.6600715

52. Nozawa M, Hara I, Matsuyama H et al (2015) Significance of baseline bone markers on disease progression and survival in hormone-sensitive prostate cancer with bone metastasis. World J Urol 33(9):1263-1268. https://doi.org/10.1007/s00345-014-1431-1
53. Okamoto T, Hatakeyama S, Narita S et al (2018) Impact of nutritional status on the prognosis of patients with metastatic hormonenaive prostate cancer: a multicenter retrospective cohort study in Japan. World J Urol. https://doi.org/10.1007/s00345-018-2590-2

54. Pan X, Zhang X, Gong J et al (2016) The expression profile and prognostic value of SPINK1 in initially diagnosed bone metastatic prostate cancer. Prostate 76(9):823-833. https://doi.org/10.1002/ pros. 23173

55. Pelger RC, Lycklama ANGA, Zwinderman AH et al (2002) The flare in alkaline phosphatase activity post-orchidectomy predicts which patient may benefit from early chemotherapy in metastatic prostate cancer. Prostate 50(2):119-124

56. Peng B, Yang C, He J (2016) Radiotherapy cannot prolong overall survival of young prostate cancer patients with bone metastases. $\mathrm{J}$ Transl Med 14:102. https://doi.org/10.1186/s12967-016-0868-y

57. Reynard JM, Peters TJ, Gillatt D (1995) Prostate-specific antigen and prognosis in patients with metastatic prostate cancera multivariable analysis of prostate cancer mortality. Br J Urol 75(4):507-515

58. Robinson D, Sandblom G, Johansson R et al (2008) Prediction of survival of metastatic prostate cancer based on early serial measurements of prostate specific antigen and alkaline phosphatase. The Journal of urology 179 (1):117-122; discussion 122-113. doi:10.1016/j.juro.2007.08.132

59. Saito T, Hara N, Kitamura Y et al (2007) Prostate-specific antigen/ prostatic acid phosphatase ratio is significant prognostic factor in patients with stage IV prostate cancer. Urology 70(4):702-705. https://doi.org/10.1016/j.urology.2007.05.019

60. Salminen EK, Kallioinen MJ, Ala-Houhala MA et al (2006) Survival markers related to bone metastases in prostate cancer. Anticancer Res 26 (6 C):4879-4884

61. Sato H, Narita S, Tsuchiya N et al (2018) Impact of early changes in serum biomarkers following androgen deprivation therapy on clinical outcomes in metastatic hormone-sensitive prostate cancer. BMC Urol 18(1):32. https://doi.org/10.1186/s12894-018-0353-4

62. Shimodaira K, Nakashima J, Nakagami Y et al (2019) Prognostic value of platelet counts in patients with metastatic prostate cancer treated with endocrine therapy. Urol J. https://doi.org/10.22037/ uj.v0i0.4735

63. Stokkel M, Zwinderman A, Zwartendijk J et al (1997) The value of pretreatment clinical and biochemical parameters in patients with newly diagnosed untreated prostate carcinoma and no indications for bone metastases on the bone scintigram. Eur J Nucl Med 24(10):1215-1220

64. Tsuchiya N, Narita S, Inoue T et al (2013) Insulin-like growth factor-1 genotypes and haplotypes influence the survival of prostate cancer patients with bone metastasis at initial diagnosis. BMC Cancer. https://doi.org/10.1186/1471-2407-13-150

65. Vesalainen S, Lipponen P, Talja M et al (1995) Biochemical parameters as prognostic factors in prostatic adenocarcinoma. Acta Oncol (Stockholm, Sweden) 34(1):53-59

66. Wang H, Fang K, Zhang J et al (2017) The significance of De Ritis (aspartate transaminase/alanine transaminase) ratio in predicting pathological outcomes and prognosis in localized prostate cancer patients. Int Urol Nephrol 49(8):1391-1398. https://doi. org/10.1007/s11255-017-1618-7

67. Yamada Y, Naruse K, Nakamura K et al (2010) Investigation of risk factors for prostate cancer patients with bone metastasis based on clinical data. Exp Therap Med 1(4):635-639. https://doi. org/10.3892/etm_00000099

68. Yashi M, Nukui A, Kurokawa S et al (2003) Elevated serum progastrin-releasing peptide (31-98) level is a predictor of short response duration after hormonal therapy in metastatic prostate cancer. Prostate 56(4):305-312. https://doi.org/10.1002/ pros. 10260 
69. Zhao J, Sun G, Liao B et al (2018) Novel nomograms for castration-resistant prostate cancer and survival outcome in patients with de novo bone metastatic prostate cancer. BJU Int 122(6):994-1002. https://doi.org/10.1111/bju.14398

70. Ren HY, Sun LL, Li HY et al (2015) Prognostic significance of serum alkaline phosphatase level in osteosarcoma: a meta-analysis of published data. Biomed Res Int 2015:160835. https://doi. org/10.1155/2015/160835
Publisher's Note Springer Nature remains neutral with regard to jurisdictional claims in published maps and institutional affiliations. 